Journal of Environmental
Analysis and Progress
ISSN: $2525-815 \mathrm{X}$

\title{
Florística, aspectos fenológicos e agrupamentos ecológicos de Bromeliaceae na Caatinga em Sergipe
}

\section{Floristics, phenological aspects and ecological groupings of Bromeliaceae in the Caatinga at Sergipe}

Larissa da Paixão Rocha ${ }^{\mathrm{a}}$, José Jailson Lima Bezerra ${ }^{\mathrm{b}}$, Ana Paula do Nascimento Prata ${ }^{\mathrm{c}}$, João Vicente Coffani-Nunes ${ }^{\mathrm{d}}$, Andressa Cristina Ribeiro Assunção ${ }^{\mathrm{c}}$, Marta Cristina Vieira Farias ${ }^{\mathrm{a}}$

${ }^{a}$ Universidade Federal de Sergipe-UFS, Departamento de Biologia, Av. Marechal Rondon, s/n, São Cristóvão, Sergipe, Brasil. CEP: 49100-000. E-mail: larissadapaixaor@hotmail.com, marta.ase@ gmail.com.

${ }^{\mathrm{b}}$ Universidade Federal de Pernambuco-UFPE, Departamento de Botânica, Av. Prof. Moraes Rego, n. 1235, Cidade Universitária, Recife, Pernambuco, Brasil. CEP: 50670-901. E-mail: josejailson.bezerra@ hotmail.com.

${ }^{\mathrm{c}}$ Universidade Federal de Alagoas-UFAL, Centro das Engenharias e Ciências Agrárias, BR-104, Km 85, s/n, Rio Largo, Alagoas, Brasil. CEP: 57100-000. E-mail: acriassuncao@gmail.com, ana.prata@ ceca.ufal.br.

${ }^{\mathrm{d}}$ Universidade Estadual Paulista-UNESP, Campus de Registro, Departamento de Engenharia de Pesca. Av. Nelson Brihi Badur, n. 430, Registro, São Paulo, Brasil. CEP: 11900-000. E-mail: jvcoffani@uol.com.br.

\section{A R T I C L E I N F O}

Recebido 19 Dez 2020

Aceito 14 Jun 2021

Publicado 13 Set 2021

\begin{abstract}
A B S T R A C T
Bromeliads represent a rich family of plants with neotropical distribution, occurring in several regions of the world. There are reports in the literature about Bromeliaceae's occurrence in dry forests, such as the Caatinga, the main biome in northeastern Brazil. Therefore, this study aimed to present information on the phenology and diversity of bromeliads occurring in Nossa Senhora da Glória, Sergipe, Brazil. Between June 2013 and June 2014, floristic analyzes were carried out to determine the genera and species of Bromeliaceae that occur in this municipality. For this purpose, ten clusters of bromeliads were selected through geographic mapping to facilitate data collection and understand the phenological behavior of species in the study area. It was found that the floristic survey contributed considerably to the increase in knowledge about the number of genera and species of Bromeliaceae in the studied location, totaling 11 species belonging to 8 genera. Tillandsia was the best-represented genus with four species. The grouping showed flowering and fruiting in different periods. During September 2013 and May 2014, the largest number of fertile individuals was found, while October 2013 had the lowest sampling rate. The ecological and phenological data found can help the management and conservation practices of bromeliads in that municipality.
\end{abstract}

Keywords: Bromeliads, checklist, biodiversity, semiarid.

\section{R E S U M O}

As bromélias representam uma rica família de plantas com distribuição neotropical, ocorrendo em várias regiões do mundo. Existem relatos na literatura sobre a ocorrência de Bromeliaceae em florestas secas, como é o caso da Caatinga, principal bioma do nordeste brasileiro. Diante disso, este estudo objetivou apresentar informações sobre a fenologia e diversidade de bromélias ocorrentes no município de Nossa Senhora da Glória, Sergipe, Brasil. Entre junho de 2013 e junho de 2014 foram realizadas análises florísticas para determinar os gêneros e espécies de Bromeliaceae que ocorrem neste município. Para esta finalidade, foram selecionados dez agrupamentos de bromélias por meio de mapeamento geográfico para facilitar a coleta de dados e entender o comportamento fenológico das espécies na área de estudo. Constatou-se que o levantamento florístico contribuiu de forma considerável para o aumento do conhecimento sobre o número de gêneros e espécies de Bromeliaceae na localidade estudada, totalizando 11 espécies pertencentes a 8 gêneros. Tillandsia foi o gênero mais bem representado com quatro espécies. Os 
agrupamentos apresentaram floração e frutificação em períodos distintos. Durante os meses de setembro de 2013 e maio de 2014 foi verificado o maior número de indivíduos férteis, enquanto o mês de outubro de 2013 teve o menor índice de amostragem. Os dados ecológicos e fenológicos encontrados podem auxiliar práticas de manejo e conservação das bromélias no referido município.

Palavras-Chave: Bromélias, checklist, biodiversidade, semiárido.

\section{Introdução}

Bromeliaceae é uma família bem diversificada incluindo ervas perenes, terrestres, epífitas ou rupícolas, com distribuição neotropical (somente uma espécie em continente africano) (Sousa \& Wanderley, 2015). A lista atualizada por Gouda e Butcher (2020) aponta o total de 3.599 espécies distribuídas em 76 gêneros e representados por oito subfamílias (Brocchinioideae, Lindmanioideae, Tillandsioideae, Hechtioideae, Navioideae, Pitcairnioideae, Puyoideae e Bromelioideae) (Givinish et al., 2011). O Brasil abriga atualmente 1.396 espécies de bromélias, sendo 1.200 endêmicas, com grande representatividade na Floresta Atlântica (Flora do Brasil, 2020). Atualmente são registrados no domínio fitogeográfico da Caatinga 132 espécies, sendo 75 endêmicas (Flora do Brasil, 2020). No estado de Sergipe ocorrem 34 espécies de bromélias distribuídas em 14 gêneros e três subfamílias, sendo que metade ocorre na região do semiárido (Sousa \& Wanderley, 2015; Flora do Brasil, 2020).

As bromélias são importantes no estabelecimento de novas espécies vegetais e na ocorrência de padrões de agrupamentos fisionomicamente diferenciados (Sampaio, Picó \& Scarano, 2005; Scarano, 2009). Devido a capacidade de acumular água, as bromélias representam importantes guildas para estabelecimento de fauna invertebrada no ambiente semiárido (Islair et al., 2015; Marteis et al., 2017). Estas espécies colonizam os ambientes tropicais e subtropicais em populações adensadas, especialmente as espécies com reprodução vegetativa clonal, cuja redução e aumento dos agrupamentos, podem ser influenciados pelas condições ambientais (Rocha, Duarte \& Waechter, 2015; Freitas et al., 2020; Barberis et al., 2020). Nesse contexto, evidencia-se a tolerância das bromélias aos ambientes rochosos e semiáridos devido a adaptações foliares de suculência; tricomas peltados e margens espinescentes contra dessecação e herbivoria; modificações da parte aérea para acesso e economia de água e nutrientes; raízes rizomatosas e metabolismo ácido crassuláceo (CAM) (Benzing, 2000; Silvestro, Zizka \& Schulte, 2014).

Essa família botânica também representa fonte de matéria prima para a população sertaneja.
Neoglaziovia variegata (Arr. Cam.) Mez, por exemplo, é vista como uma importante espécie produtora de fibras, sendo amplamente utilizada para a confecção artesanal de cordas e barbantes (Lima, 1989). Bromelia laciniosa Mart. ex Schult. \& Schult. f. pode ser utilizada na alimentação de animais e até mesmo na nutrição dos seres humanos, onde é possível extrair uma massa da base de suas folhas da qual se fabrica um tipo de pão (Angelim et al., 2007). Pode ser considerada uma espécie-chave para a vegetação da Caatinga em função das suas interações ecológicas (Santo, Maciel \& Siqueira Filho, 2012), além disso também pode ser utilizada como cerca viva em propriedades rurais ou no entorno de rodovias para evitar a erosão.

As florestas secas, tais como a Caatinga, são os biomas menos estudados na literatura (Sunderland et al., 2015), especialmente quando se trata do estrato herbáceo, que representa grande importância para a biodiversidade das áreas secas (Moro et al., 2015). A Caatinga destaca-se como a maior ecorregião semiárida do continente Sulamericano (Moro et al., 2016), sua diversificada vegetação inclui formações abertas com domínio de algumas famílias, incluindo-se Bromeliaceae (Velloso, Sampaio \& Pareyn, 2002). Após o estudo de Sousa \& Wanderley (2015), o levantamento florístico mais atual sobre bromélias na Caatinga foi realizado no Rio Grande do Norte (Tomaz \& Versieux, 2019).

No estado de Sergipe, que tem cerca de $50 \%$ do território na Caatinga (IBGE, 2004), os esforços de estudos com comunidades herbáceas continuam a ser necessários (Oliveira, Prata \& Ferreira, 2013; Gouveia et al., 2015; Moro et al., 2015; Oliveira, Prata \& Pinto, 2018) frente as limitações de coleta devido ao acesso, a escassez de taxonomistas no estado e a conversão da vegetação nativa em pastagens e práticas agrícolas inadequadas (Gouveia et al., 2015). Os municípios do estado com maior amostragem da vegetação são Canindé de São Francisco (Silva, Prata \& Mello, 2013; Silva, Prata \& Mello, 2016), Poço Redondo (Machado, Prata \& Mello, 2012; Silva, Prata \& Mello, 2013; Silva, Prata \& Mello, 2016), Poço Verde (Ferreira, Prata \& Mello, 2013; Oliveira, Prata \& Pinto, 2018) e Porto da Folha (Oliveira, Prata \& Ferreira, 2013; Gouveia et al., 2015). 
Nossa Senhora da Glória faz limite com Porto da Folha, um dos municípios sergipanos mais bem amostrado e que possui remanescentes de Caatinga bastante antropizados, principalmente em decorrência da exploração da pecuária. Devido a relevante ocorrência de bromélias nas áreas de Caatinga do território, objetivou-se apresentar informações sobre fenologia, agrupamentos ecológicos e diversidade de bromélias ocorrentes no município de Nossa Senhora da Glória, Sergipe.

\section{Material e Métodos}

Caracterização da área de estudo
Este estudo foi desenvolvido no município de Nossa Senhora da Glória, no Assentamento Nossa Senhora Aparecida, que se encontra localizado a aproximadamente $17 \mathrm{~km}$ após a entrada da sede do município $\left(10^{\circ} 06^{\prime} 12.64\right.$ " S / $37^{\circ} 25^{\prime} 11.35^{\prime}$ W). O município está localizado na região noroeste do Estado de Sergipe, limitando-se com os municípios de Monte Alegre de Sergipe e Porto da Folha a norte; Gararu, Gracho Cardoso e Feira Nova a leste; Nossa Senhora Aparecida e Carira ao sul; e o Estado da Bahia a oeste (Figura $1)$.

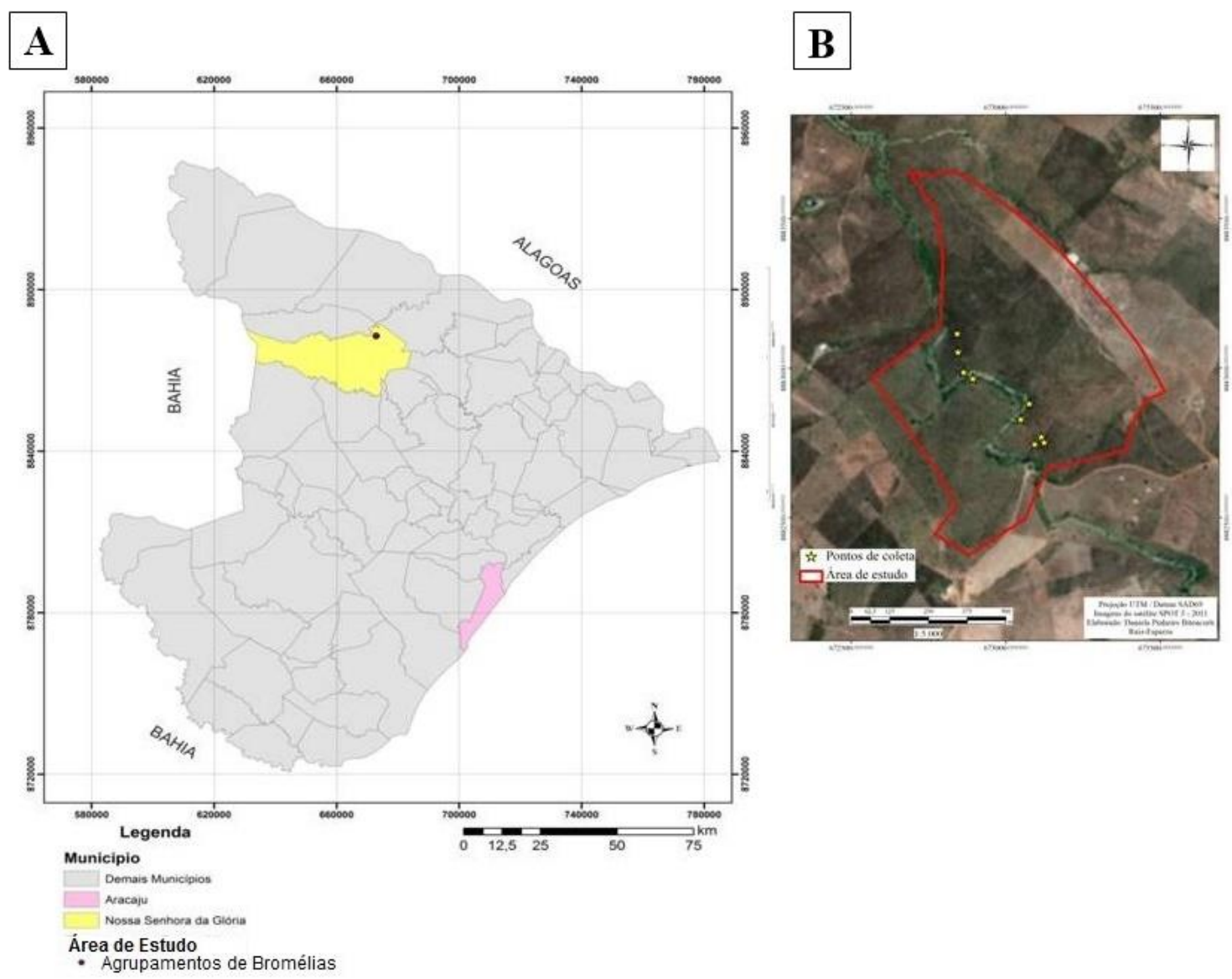

Figura 1. Estado de Sergipe. A. Área de estudo; B. Pontos de coleta. Fonte: SEMARH/SRH (2011).

O município faz parte do Polígono das Secas, na Região Climática do Semiárido (clima do tipo megatérmico semiárido), com temperatura média anual de aproximadamente $24^{\circ} \mathrm{C}$, precipitação pluviométrica média de aproximadamente $702 \mathrm{~mm}$, com período chuvoso compreendido entre os meses de março e agosto. $\mathrm{O}$ relevo é caracterizado por uma superfície pediplanada e dissecada, com formas do tipo colina e tabuleiros (Bomfim, Costa \& Benvenuti, 2002). Os solos que predominam no município são: Luvissolos, Neossolos Litólicos e Planossolos (EMBRAPA, 2006; Carvalho, 2012).

\section{Análise florística}

Inicialmente, foi realizada uma análise do acervo do Herbário da Universidade Federal de Sergipe (ASE) para saber o número de espécies registrados para o município de Nossa Senhora da Glória.

Posteriormente, as espécies coletadas nas visitas a campo foram herborizadas de acordo com Mori et al. (1985) e o voucher depositado no Herbário da UFS (ASE). As coletas foram realizadas mensalmente durante o período de junho de 2013 a junho de 2014, por meio de caminhadas aleatórias para verificação das espécies de bromélias ocorrentes na área de estudo. 
A identificação do material botânico foi realizada mediante consultas a literatura disponível (Sousa \& Wanderley, 2000; Dutra, Teófilo \& Medeiros Filho, 2010; Forzza, 2005; Silva \& Gomes, 2003; Pontes, 2005; Wanderley, 1990; Bourscheid, 2008). Além disso, algumas espécies foram comparadas com os vouchers depositados no acervo do Herbário ASE e identificadas por especialistas na família.

\section{Agrupamentos ecológicos}

De acordo com a análise geral da área de estudo, foram determinados pontos onde se concentravam as principais populações de bromélias. Estes pontos estratégicos foram classificados como "agrupamentos", escolhidos na localidade onde persistia o maior número de indivíduos da mesma espécie. Como ponto de referência utilizou-se o Riacho do Chico (RC) localizado no município de Nossa Senhora da Glória, Sergipe, Brasil. No total foram selecionados 10 agrupamentos de bromélias com base no mapeamento geográfico da localidade. Este procedimento foi realizado por meio dos registros de coordenadas geográficas determinadas com GPS (Garmin-Dakota 10) para facilitar as análises mensais dos agrupamentos durante $\mathrm{o}$ período de execução do trabalho.

\section{Análise fenológica}

Para atender o objetivo do estudo, foram levantadas informações sobre o período reprodutivo das espécies presentes na área de estudo. De acordo com as análises mensais, todos os registros eram documentados para identificar quando as espécies de Bromeliaceae apresentavam botões florais, flores, flores senescentes, frutos imaturos, frutos maduros e frutos senescentes. Dessa forma, o agrupamento de bromélias era considerado fértil quando ao menos um indivíduo do agrupamento estava florido ou frutificado. Caso não houvesse nenhum sinal de fase reprodutiva nos agrupamentos durante os meses de coleta, estes eram considerados estéreis.

\section{Resultados e Discussão \\ Análise florística}

Os dados coletados no Herbário ASE, até o ano de 2012 apontou a ocorrência de três gêneros e cinco espécies (Aechmea aquilega (Salisb.) Griseb., Hohenbergia catingae Ule, Tillandsia gardneri Lindl., T. loliacea Mart. ex Schult. \& Schult.f. e $T$. polystachia L.) de Bromeliaceae no município de Nossa Senhora da Glória. Após o levantamento florístico realizado durante o desenvolvimento deste estudo, o conhecimento sobre o número de gêneros e espécies aumentou consideravelmente, tendo em vista que foram registrados um total de 11 espécies pertencentes a 8 gêneros (Tabela 1). Desta forma, o município supracitado detém $31,6 \%$ das espécies de bromélias do estado de Sergipe.

Tabela 1. Listagem de espécies de Bromeliaceae coletadas em Nossa Senhora da Glória, Sergipe, Brasil. Fonte: Rocha et al. (2015).

\begin{tabular}{llll}
\hline Espécies & Hábito (*) & Coletor & ASE \\
\hline Aechmea aquilega (Salisb.) Griseb. & Terrestre & Prata, A.P. & 28351 \\
Bromelia laciniosa Mart. ex Schult. \& Schult.f. & Terrestre & Rocha, L.P. & 29498 \\
Cryptantus sergipensis I.Ramírez & Terrestre & Prata, A.P. & 28341 \\
Encholirium spectabile Mart. ex Schult. \& Schult.f. & Rupícola & Rocha, L.P. & 30279 \\
Hohenbergia catingae Ule. & Terrestre & Rocha, L.P. & 29503 \\
Neoglaziovia variegata (Arr. Cam.) Mez & Terrestre & Prata, A.P. & 28350 \\
Orthophytum maracasense L.B.Sm & Rupícola & Rocha, L.P. & $(* * *)$ \\
Tillandsia loliacea Mart. ex Schult. \& Schult.f. & Epífita & Prata, A.P. & 28345 \\
Tillandsia polystachia (L.) L. & Epífita & Rocha, L.P. & 29504 \\
Tillandsia recurvata L.) L. & Epífita & Prata, A.P. & 26360 \\
Tillandsia streptocarpa Baker & Epífita & Prata, A.P. & 28356 \\
\hline
\end{tabular}

(*) hábito de acordo com a literatura (Sousa \& Wanderley, 2000; Dutra, Teófilo \& Medeiros Filho, 2010; Forzza, 2005; Silva \& Gomes, 2003; Pontes, 2005; Wanderley, 1990; Bourscheid, 2008), (***) sem número de registro.

Tilandsia foi o gênero mais frequente na amostragem realizada, com um total de quatro espécies: T. loliacea, $T$. polystachia, $T$. recurvata e T. streptocarpa (Tabela 1). Estes dados eram esperados, tendo em vista que, segundo Gomes \& Silva (2013), as espécies de maior distribuição no
Brasil pertencem aos gêneros Aechmea e Tillandsia. A ampla ocorrência dessas espécies é também corroborada por Tomaz \& Versieux (2019) no Rio Grande do Norte, onde foi registrada a ocorrência de 12 espécies de Tillandsia seguido por três espécies de Aechmea e de Hohenbergia. 
Deus \& Bourscheid (2016) também evidenciaram que no Parque Estadual Rio Canoas em Campos Novos, Santa Catarina, o gênero Tillandsia foi o mais frequente na amostra do fragmento florestal estudado, representando $42,85 \%$ das espécies levantadas. Ainda no estudo de Büneker \& Witeck-Neto (2016), Tillandsia também foi o gênero melhor representado com nove espécies registradas num levantamento de bromélias realizado no curso médio do Rio Toropi, Rio Grande do Sul. Assim, fica evidente que, tanto no Nordeste do Brasil, como no Sul, os estudos realizados apontam que Tillandsia é o gênero melhor representado.

É importante ressaltar que o desenvolvimento de pesquisas que buscam descrever a ocorrência e o conhecimento das espécies em uma região contribui para uma série de outros estudos, como programas conservacionistas e estudos em biogeografia da distribuição de plantas de Caatinga (Versieux, Tomaz \& Jardim, 2013).

Agrupamentos de espécies de Bromeliaceae no município de Nossa Senhora da Glória, Sergipe, Brasil

Dentre os 10 agrupamentos (Figura 2), apenas o agrupamento 7 se encontra constituído por duas espécies de Bromeliaceae. O restante é formado por apenas uma espécie, provavelmente formada por desenvolvimento clonal. A seguir, estão descritas as características gerais de cada agrupamento estudado.

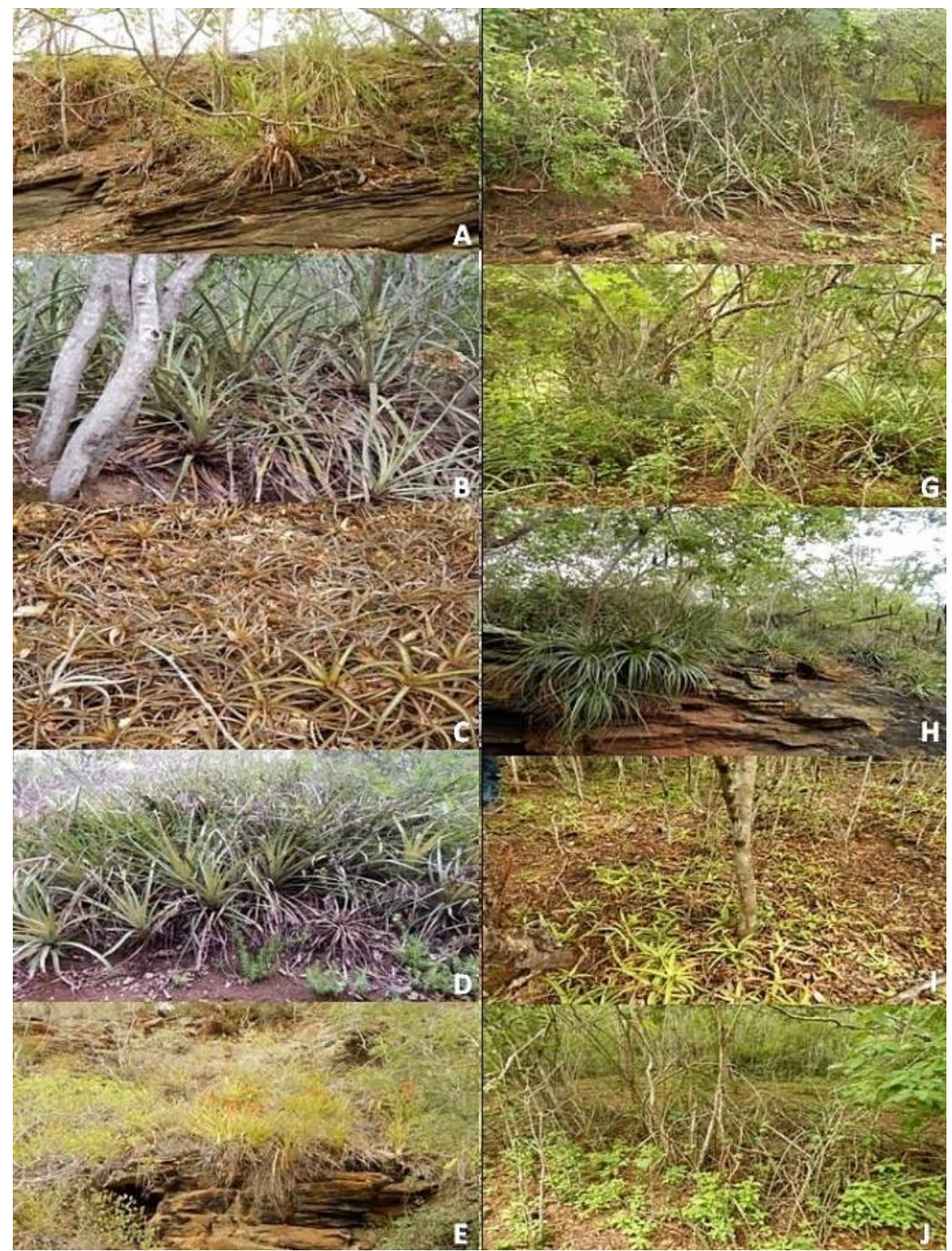

Figura 2. Visão geral dos agrupamentos de bromélias do Assentamento Nossa Senhora Aparecida, Nossa Senhora da Glória, Sergipe, Brasil. A. Agrupamento 1; B. Agrupamento 2; C. Agrupamento 3; D. Agrupamento 4; E. Agrupamento 5; F. Agrupamento 6; G. Agrupamento 7; H. Agrupamento 8; I. Agrupamento 9; J. Agrupamento 10. Fonte: Rocha et al. (2014). 
O agrupamento $1 \quad\left(10^{\circ} 06^{\prime} 11,0^{\prime \prime} \quad \mathrm{S} /\right.$ $37^{\circ} 25^{\prime} 12,4^{\prime \prime} \mathrm{W}$ ) localiza-se a $2,50 \mathrm{~m}$ do RC. Este agrupamento é constituído pela espécie Aechmea aquilega (Salisb.) Griseb. (macambira-de-boi). Apresenta indivíduos instalados em uma parede rochosa com 3,0 m de altura e largura de $2,90 \mathrm{~m}$. A área do entorno apresenta um solo desnudo, sem a presença de serapilheira e a sua vegetação é composta basicamente por árvores de Poincianella pyramidalis (Tul.) L.P. Queiroz (catingueira).

O agrupamento $2 \quad\left(10^{\circ} 06^{\prime} 11,6^{\prime \prime} \mathrm{S} /\right.$ $37^{\circ} 25^{\prime} 12,1^{\prime \prime} \mathrm{W}$ ) localiza-se a $1,10 \mathrm{~m}$ do RC. Este agrupamento é constituído pela espécie Bromelia laciniosa Mart. ex Schult. \& Schult.f (macambira). Agrupamento sobre solo arenoso, com a uma extensão de 5,60 e largura de 5,80 m. A área do entorno apresenta um solo recoberto por serapilheira e a sua vegetação é composta basicamente por árvores de P. pyramidalis (Tul.) L.P. Queiroz (catingueira).

O agrupamento $3 \quad\left(10^{\circ} 06^{\prime} 11,8^{\prime \prime} \quad \mathrm{S} /\right.$ $37^{\circ} 25^{\prime} 13,1^{\prime \prime}$ W) localiza-se a $13 \mathrm{~m}$ do RC. Situado no interior da mata, é um dos lugares mais sombreados da área de estudo. Este agrupamento, sobre solo arenoso, é constituído por Cryptantus sergipensis I. Ramírez, alcançando uma extensão de $3,10 \mathrm{~m}$ e largura de $2,70 \mathrm{~m}$. A área do entorno apresenta um solo recoberto por serapilheira e a sua vegetação é composta, basicamente, por árvores de P. pyramidalis (Tul.) L.P. Queiroz (catingueira).

O agrupamento $4 \quad\left(10^{\circ} 06^{\prime} 09,1^{\prime \prime} \quad \mathrm{S} \quad /\right.$ $\left.37^{\circ} 25^{\prime} 14,6^{\prime \prime} \mathrm{W}\right)$ localiza-se a $0,70 \mathrm{~cm}$ do $\mathrm{RC}$, em uma ilha isolada. Este agrupamento é constituído pela espécie B. laciniosa Mart. ex Schult. \& Schult.f (macambira). Apresenta indivíduos sobre solo arenoso, com o aglomerado alcançando uma extensão de 5,60 m e largura de 3,80 m. A área do entorno apresenta um solo recoberto por serapilheira e a sua vegetação é composta basicamente por árvores de Cenostigma pyramidale (Tul.) E. Gagnon \& G.P. Lewis var pyramidale (catingueira). Durante a execução do projeto, nos meses de janeiro e fevereiro, em função do volume elevado de chuvas, foi observado a mudança temporária do curso do rio, com o aumento do nível de água o agrupamento 4 foi atingido, causando $\mathrm{o}$ arrasto parcial de indivíduos desse agrupamento.

O agrupamento $5 \quad\left(10^{\circ} 06^{\prime} 09,1^{\prime \prime} \quad \mathrm{S} /\right.$ $37^{\circ} 25^{\prime} 14,6^{\prime \prime} \mathrm{W}$ ) localiza-se a 9,70 m do RC. Este agrupamento é constituído por A. aquilega (Salisb.) Griseb. (macambira-de-boi). Apresenta indivíduos agrupados em parede rochosa, alcançando uma altura de 4,60 m e largura de 3,0 m. A área do entorno apresenta uma vegetação formada por herbáceas.
O agrupamento $6 \quad\left(10^{\circ} 06^{\prime} 07,4^{\prime \prime} \quad \mathrm{S} \quad /\right.$ $\left.37^{\circ} 25^{\prime} 13,7^{\prime \prime} \mathrm{W}\right)$ localiza-se a $1,20 \mathrm{~m}$ do RC. Este agrupamento é constituído por B. laciniosa Mart. ex Schult. \& Schult.f (macambira). Apresenta indivíduos em solo arenoso, alcançando uma extensão de 9,70 e largura de 4,0 m. A área do entorno apresenta um solo exposto, sem a presença de serapilheira e a vegetação do entorno é composta basicamente por árvores de Maytenus rigida Mart (bom-nome) e cactáceas. O grande adensamento de bromélias visto neste agrupamento inviabilizou a visualização do solo entre as bromélias, o que acarreta uma proteção do solo.

O agrupamento $7 \quad\left(10^{\circ} 06^{\prime} 04,7^{\prime \prime} \mathrm{S} \quad /\right.$ $\left.37^{\circ} 25^{\prime} 19,7^{\prime \prime} \mathrm{W}\right)$ localiza-se a $4,0 \mathrm{~m}$ do RC. Este agrupamento é constituído pelas espécies $B$. laciniosa Mart. ex Schult. \& Schult.f (macambira) e Hohenbergia catingae Ule. (macambira-roxa). Também sobre solo arenoso, alcançando uma extensão de 10,20 m e largura de 5,50 m. A área do entorno apresenta solo com serapilheira e a vegetação é composta basicamente de herbáceas, Pilosocereus catingicola (Gürke) Byles \& Rowley (facheiro) e Tacinga palmadora (Britton \& Rose) N.P.Taylor \& Stuppy (quipá), espécies de Verbenaceae não identificadas, árvores de $P$. pyramidalis (Tul.) L.P. Queiroz (catingueira) e Guapira noxia (Netto) Lundell (joão-mole).

O agrupamento $8 \quad\left(10^{\circ} 06^{\prime} 04,0^{\prime \prime} \quad \mathrm{S} \quad /\right.$ $37^{\circ} 25^{\prime} 20,7^{\prime \prime} \mathrm{W}$ ) localiza-se a $4,50 \mathrm{~m}$ do RC. Este agrupamento é constituído por Encholirium spctabile Mart. ex Schult. \& Schult.f. (macambirade-flecha). Apresenta indivíduos aglomerados em parede rochosa, alcançando uma altura de $3,40 \mathrm{~m}$ e largura de $12,10 \mathrm{~m}$. A área do entorno apresenta um solo com a presença de serapilheira e a vegetação constituída de herbáceas Melocactus zehntneri (Britton \& Rose) Luetzelb. (cabeça de frade), Tacinga palmadora (Britton \& Rose) N.P. Taylor \& Stuppy (quipá), e árvore Anadenanthera colubrina (vell.) Brenan (angico).

O agrupamento $9 \quad\left(10^{\circ} 06^{\prime} 01,8^{\prime \prime} \quad \mathrm{S} /\right.$ $37^{\circ} 25^{\prime} 21,3^{\prime \prime} \mathrm{W}$ ) localiza-se a 7,0 m do RC, na parte interna da área de estudo, uma área sombreada. Constituído por Cryptantus sergipensis I. Ramírez, alcançando uma extensão de $6,0 \mathrm{~m}$ e largura e de $5,0 \mathrm{~m}$ sobre o solo com de serapilheira no entorno. Nessa área encontram-se árvores de $P$. pyramidalis (Tul.) L.P. Queiroz (catingueira) e Maytenus rigida Mart (bom-nome).

O agrupamento $10 \quad\left(10^{\circ} 05^{\prime} 59,8^{\prime \prime} \quad \mathrm{S} /\right.$ $37^{\circ} 25^{\prime} 21,4^{\prime \prime} \mathrm{W}$ ) localiza-se a $20,0 \mathrm{~m}$ do RC. Este agrupamento é o único constituído somente por $N$. variegata (Arr. Cam.) Mez. (caroá), alcançando uma extensão de 3,70 m e largura de 4,0 m. A área do entorno apresenta um solo com serapilheira e árvores de P. pyramidalis (Tul.) L.P. Queiroz 
(catingueira) e herbáceas. Durante as observações foi verificado que a espécie $N$. variegata se mantinha distante da borda do riacho, pois são, preferencialmente, de lugares menos úmidos.

Durante as épocas de seca que ocorreu de setembro de 2013 a março de 2014 foi observado que as árvores perdem as suas folhas. Os agrupamentos que estão localizados em paredão rochoso (agrupamentos 1, 5 e 8 ) sempre recebem maior incidência de luz. $\mathrm{O}$ agrupamento quatro, por estar mais exposto ao sol, devido a ocorrência de menos árvores e arbustos próximos a ela recebe luz direta.

Segundo Versieux et al. (2018), a vegetação mais aberta da savana e florestas sazonais secas, juntamente com o alto grau de afloramento (especialmente calcário e arenito), provavelmente são mais propícios ao hábito de vida da maioria das Bromelioideae e Pitcairnioideae. No presente estudo constatou-se a presença das duas subfamílias com grande predominância das Bromelioideae com cinco espécies, corroborando o autor supracitado.

\section{Análise fenológica}

Os agrupamentos apresentaram floração e frutificação em meses distintos. Durante os meses de setembro de 2013 e maio de 2014 foi verificado o maior número de indivíduos férteis nos agrupamentos, enquanto o mês de outubro de 2013 teve a menor ocorrência de agrupamentos férteis. Mas, mesmo assim, houve indivíduos férteis ao longo de quase todo o período de observação, com uma tendência de uma maior frequência nos períodos de chuva (Tabela 2).

Tabela 2. Amostragem dos agrupamentos de espécies de Bromeliaceae e suas respectivas fases fenológicas observadas entre os anos de 2013 e 2014. Fonte: Rocha et al. (2015).

\begin{tabular}{|c|c|c|c|c|c|c|c|c|c|c|c|c|}
\hline \multirow{3}{*}{ Agrupamentos } & \multicolumn{6}{|c|}{ Meses / Ano 2013} & \multicolumn{6}{|c|}{ Meses / Ano 2014} \\
\hline & Jun & Ago & Set & Out & Nov & Dez & Jan & Fev & Mar & Abr & Mai & Jun \\
\hline & \multicolumn{2}{|c|}{ Chuva } & \multicolumn{6}{|c|}{ Seco } & \multicolumn{4}{|c|}{ Chuva } \\
\hline 1 & $\Delta$ & $\Delta$ & $\Delta$ & $\Delta$ & $\begin{array}{ll}-- \\
--\end{array}$ & $\Delta$ & $\begin{array}{l}-- \\
--\end{array}$ & $\Delta$ & $\Delta$ & $\Delta$ & $\boldsymbol{\Delta}$ & $\Delta$ \\
\hline 2 & $\Delta$ & $\boldsymbol{\Delta}$ & $\boldsymbol{\Delta}$ & $\boldsymbol{\Delta}$ & --- & $\Delta$ & --- & $\Delta$ & $\Delta$ & $\Delta$ & $\Delta$ & $\Delta$ \\
\hline 3 & $\boldsymbol{\Delta}$ & $\Delta$ & $\Delta$ & $\Delta$ & --- & $\Delta$ & --- & $\Delta$ & $\Delta$ & $\Delta$ & $\Delta$ & $\Delta$ \\
\hline 4 & $\Delta$ & $\Delta$ & $\boldsymbol{\Delta}$ & $\Delta$ & --- & $\Delta$ & --- & $\Delta$ & $\Delta$ & $\Delta$ & $\Delta$ & $\Delta$ \\
\hline 5 & $\mathbf{\Delta}$ & $\boldsymbol{\Delta}$ & $\boldsymbol{\Delta}$ & $\Delta$ & --- & $\mathbf{\Delta}$ & --- & $\Delta$ & $\boldsymbol{\Delta}$ & $\Delta$ & $\boldsymbol{\Delta}$ & $\mathbf{\Delta}$ \\
\hline 6 & $\Delta$ & $\Delta$ & $\Delta$ & $\Delta$ & --- & $\Delta$ & --- & $\Delta$ & $\Delta$ & $\Delta$ & $\Delta$ & $\Delta$ \\
\hline 7 & $\Delta$ & $\Delta$ & $\Delta$ & $\Delta$ & --- & $\Delta$ & --- & $\Delta$ & $\Delta$ & $\Delta$ & $\Delta$ & $\Delta$ \\
\hline 8 & $\Delta$ & $\Delta$ & $\Delta$ & $\Delta$ & --- & $\Delta$ & --- & $\Delta$ & $\Delta$ & $\Delta$ & $\boldsymbol{\Delta}$ & $\Delta$ \\
\hline 9 & $\Delta$ & $\Delta$ & $\Delta$ & $\Delta$ & -- & $\Delta$ & --- & $\Delta$ & $\Delta$ & $\Delta$ & $\mathbf{\Delta}$ & $\Delta$ \\
\hline 10 & $\Delta$ & $\Delta$ & $\Delta$ & $\Delta$ & --- & $\Delta$ & --- & $\Delta$ & $\Delta$ & $\Delta$ & $\Delta$ & $\Delta$ \\
\hline
\end{tabular}

Fase reprodutiva (espécies com botões florais, flores ou frutos); $\Delta$ Fase estéril.

Por se tratar de espécies distintas, os indivíduos pertencentes a cada agrupamento possuem época de floração e frutificação diferenciadas. Deste modo, observou-se que a espécie A. aquilega (pertencente aos agrupamentos 1 e 5) floresceu e/ou frutificou em todos os meses avaliados, exceto em outubro de 2013. Silva, Wanderley \& Melo (2018) registraram que a floração de A. aquilega no estado da Paraíba, região nordeste do Brasil, acontece em novembro e dezembro, enquanto o período de frutificação ocorre em julho. Além disso, A. aquilega demonstrou-se uma espécie importante para o ambiente estudado em função de apresentar alguma fase reprodutiva ao longo do ano, podendo ser uma fonte de alimento para diferentes grupos de animais (Tabela 3).

Tabela 3. Amostragem das espécies e suas respectivas fases fenológicas. $1=$ Aechmea aquilega $2=$ Bromelia laciniosa; 3 = Crypthantus sergipensis; $4=$ Encholirium spectabile $; 5=$ Hohenbergia catingae $; 6=$ Neoglaziovia variegata. Fonte: Rocha et al. (2015).

\begin{tabular}{|c|c|c|c|c|c|c|c|c|c|c|c|c|c|}
\hline \multirow{3}{*}{ Espécies } & \multicolumn{7}{|c|}{ Meses / Ano 2013} & \multicolumn{6}{|c|}{ Meses / Ano 2014} \\
\hline & Jun & Jul & Ago & Set & Out & Nov & Dez & Jan & Fev & Mar & $\mathbf{A b r}$ & Mai & Jun \\
\hline & & Chuv & & & & & & & & & Cht & & \\
\hline 1 & $\Delta \uparrow$ & --- & 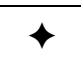 & $\Delta \leftarrow$ & $\mathrm{O}$ & --- & O & --- & $\Delta$ & $\Delta \uparrow$ & $\diamond \hookrightarrow$ & $\diamond \downarrow$ & $\square \triangleleft$ \\
\hline 2 & 0 & --- & $\square \triangleleft$ & $\diamond \triangleleft$ & $\square$ & --- & 0 & --- & 0 & 0 & 0 & 0 & 0 \\
\hline 3 & $\Delta$ & --- & O & 0 & O & --- & 0 & --- & 0 & 0 & O & $\mathbf{O A}$ & $\mathbf{\Delta}$ \\
\hline
\end{tabular}




\begin{tabular}{l|lll|llllll|llll}
4 & 0 & --- & 0 & 0 & 0 & --- & $\Delta \Delta$ & -- & 0 & 0 & 0 & $\diamond$ & 0 \\
5 & 0 & --- & 0 & 0 & 0 & -- & 0 & --- & 0 & 0 & 0 & 0 & 0 \\
6 & 0 & --- & 0 & 0 & 0 & --- & 0 & --- & 0 & 0 & 0 & 0 & 0 \\
\hline
\end{tabular}

--- Não analisado; $\bigcirc$ Estéril; $\bigcirc$ Botões florais; $\Delta$ Flores; $\square$ Frutos imaturos; $\diamond$ Frutos maduros; $\downarrow$ Frutos senescentes.

Por meio da análise de B. laciniosa (pertencente aos agrupamentos 2, 4, 6 e 7) foi possível constatar que a espécie permaneceu estéril durante todo o período de investigação em 2014 sem evidências de fase reprodutiva. Em estudo realizado por Santo, Maciel \& Siqueira Filho (2012), entre novembro de 2005 e março de 2007, no Município de Afrânio, Pernambuco, Brasil, foi verificado que nas parcelas inventariadas foram registrados 246 indivíduos de $B$. laciniosa em floração, os quais, juntos, produziram, em média, 13.284 flores.

Observou-se em Crypthantus sergipensis (pertencente aos agrupamentos 3 e 9) que suas únicas fases fenológicas presentes foram flores e botões florais, o qual foi constatado apenas em junho de 2013 e, posteriormente, em maio e junho de 2014, durante os períodos mais chuvosos.

Por meio da avaliação de Encholirium spectabile (agrupamento 8) foi possível constatar que durante todos os meses analisados ela esteve estéril, exceto em dezembro de 2013 e maio de 2014, tendo em vista que foram observados espécimes em período reprodutivo com a maioria de seus frutos expostos, não apresentando uma correlação clara com o período de chuva ou seca (Tabela 2). Diferentemente dos dados observados neste estudo, Queiroz et al. (2016) observaram que o florescimento de E. spectabile ocorreu, principalmente, entre junho e setembro e durou até outubro, período que coincide com as últimas chuvas (junho a julho) e com parte da estação seca na Caatinga.

Verificou-se ainda que a espécie $N$. variegata (pertencente ao agrupamento 10), apresentou seu período fértil apenas em setembro de 2013, onde foram observados flores e frutos imaturos, ou seja, no período de seca, contrariando o exposto por Pereira e Quirino (2008), que constatou a florada de $N$. variegata após a estação seca em uma região da Caatinga paraibana (Tabela $3)$.

Algumas espécies como A. aquilega, $B$. lacionosa, E. spectabile e $N$. variegata tiveram comportamento fenológico diferenciado em relação ao reportado na literatura. Isso pode estar relacionado ao fato dos agrupamentos estarem próximos ao rio e, portanto, estarem em um ambiente com maior oferta de água e umidade ao longo do ano. Desta forma, novos estudos comparando agrupamentos com proximidades distintas do rio seriam necessários para discutir a possível interferência do mesmo na fenologia das espécies de Caatinga.

\section{Conclusão}

Os esforços de amostragem de bromélias em escala local ampliaram o conhecimento sobre a diversidade destas espécies no município de Nossa Senhora da Glória em Sergipe. Além disso, os estudos dos agrupamentos facilitam a compreensão dos mosaicos de Bromeliaceae existentes na região de Caatinga e sua importância para o ambiente. Assim, dados ecológicos e fenológicos encontrados podem auxiliar práticas de manejo e conservação das bromélias no município. Apesar de $H$. caatingae ter permanecido estéril ao longo do estudo, A. aquilega esteve fértil durante todo o período de análise. Isso salienta a sua possível importância como recurso alimentar para as espécies da região. É necessário o desenvolvimento de novos estudos para avaliar se a proximidade de corpos d'agua pode interferir nas fases fenológicas das bromélias de Caatinga.

\section{Agradecimentos}

Ao Conselho Nacional de Desenvolvimento Científico e Tecnológico CNPq.

\section{Referências}

Angelim, A. E. S.; Moraes, J. P. S.; Silva, J. A. B.; Gonçalves-Gervásio, R. C. R. 2007. Germinação e Aspectos morfológicos de plantas de macambira (Bromelia laciniosa), encontradas na Região do Vale do São Francisco. Revista Brasileira de Biociências, 5, 1065-1067.

Barberis, I. M.; Klekailo, G.; Albertengo, J.; Cárcamo, J. I.; Cárcamo, J. M.; Galetti, L. 2020. Ramet demography of Aechmea distichantha (Bromeliaceae) in two contrasting years in the understory and open áreas of a South American xerophytic forest. Rodriguésia, $71, \quad 1-11$. https://doi.org/10.1590/21757860202071014

Benzing, D. V. 2000. Bromeliaceae: Profile of an adaptive radiation. United Kingdom at the University Press, Cambridge. 
Bomfim, L. F. C.; Costa, I. V. G.; Benvenuti, S. M. P. 2002. Projeto cadastro da infra-estrutura hídrica do nordeste: estado de Sergipe. diagnóstico do município de Nossa Senhora da Glória - Aracaju: CPRM. pp. 1-22. Disponível em: http://rigeo.cprm.gov.br/xmlui/handle/doc/25 13. Acesso em: 06 de jun. 2020.

Bourscheid, K. 2008. Levantamento das Bromeliaceae Juss. da Fazenda Acaraú, Bertioga, São Paulo. Dissertação de Mestrado, Universidade Federal de Santa Catarina, Santa Catarina, Brasil. 82p.

Büneker, H. M.; Witeck-Neto L. 2016. Levantamento de Bromeliaceae na região do curso médio do rio Toropi, Rio Grande do Sul, Brasil. Balduinia, 52, 01-14. https://doi.org/10.5902/2358198022371

Carvalho, M. E. S. 2012. Vulnerabilidade hídrica na bacia sergipana do rio Vaza Barris. Raega, 25, 186-217.

Deus, J. J. C.; Bourscheid, K. 2016. Levantamento florístico de Bromeliaceae no Parque Estadual Rio Canoas em Campos Novos, Santa Catarina. Unoesc \& Ciência-ACBS, 7, 83-88.

Dutra, A. S.; Teófilo, E. M.; Medeiros Filho, S. 2010. Germinação de sementes de macambira (Bromelia laciniosa Mart. ex Schult). Revista Caatinga, 23, 12-17.

EMBRAPA. 2006. Empresa Brasileira de Pesquisa Agropecuária, Centro Nacional de Pesquisa de Solos. Sistema de Classificação Brasileiro de Classificação de Solos. 2. ed. 1-306.

Ferreira, E. V. R.; Prata, A. P. N.; Mello, A. A. 2013. Floristic list from a caatinga remnant in Poço Verde, Sergipe, Brazil. Check List, 9, 1354-1360.

Flora do Brasil 2020 em construção. Bromeliaceae. Jardim Botânico do Rio de Janeiro. Disponível em: http://floradobrasil.jbrj.gov.br/reflora/florado brasil/FB66>. Acesso em: 05 de jun. 2020.

Forzza, R. C. 2005. Revisão taxonômica de Encholirium Mart. Ex Schult. \& Schult. F. (Pitcairniodeae -Bromeliaceae). Boletim de Botânica, 23, 1-49. https://doi.org/10.11606/issn.23169052.v23i1p1-49

Freitas, L.; Ribeiro, P. C.; Cancio, A. S.; Machado, M. A.; Sampaio, M. C.; Forzza, R. C.; Viccini, L. F. 2020. Population demography, genetic variation and reproductive biology of two rare and endangered Neoregelia species (Bromeliaceae). Botanical Journal of the Linnean Society, 192, 787-802. https://doi.org/10.1093/botlinnean/boz110
Givnish, T. J.; Barfuss, M. H.; Van Ee, B.; Riina, R.; Schulte, K.; Horres, R. et al. 2011. Phylogeny, adaptive radiation, and historical bio-geography in Bromeliaceae: Insights from an eight-locus plastid phylogeny. American Journal of Botany, 98, 872-895. https://doi.org/10.3732/ajb.1000059

Gomes, J. M. L.; Silva, N. N. F. 2013. Bromeliaceae das restingas do Estado do Espírito Santo, Brasil. Natureza on line, 11, 79-89.

Gouda, E. J.; Butcher, D. 2020. A list of accepted Bromeliaceae names, University Botanic Gardens, Utrecht. Disponível em: http://bromeliad.nl/bromNames/. Acesso em: 14 de abr. 2020.

Gouveia, S. F.; Prata, A. P.; Ladim, M.; Farias, M. C. V. 2015. Distribuição espacial da diversidade florística de Sergipe: síntese do conhecimento atual. In: Prata, A. P. N. et al. Flora de Sergipe. Editora Criação, pp. Xxx$\mathrm{xxx}$.

IBGE. 2004. Instituto Brasileiro de Geografia e Estatistica. Mapa de Biomas do Brasil, primeira aproximação. Disponível em: http://www.ibge.gov.br. Acesso em: 15 de abr. 2020.

Islair, P.; Carvalho, K. S.; Ferreira, F. C.; Zina, J. 2015. Bromeliads in Caatinga: an oasis for invertebrates. Revista Biotemas, 28, 67-77. https://doi.org/10.5007/21757925.2015v28n1p67

Lima, D. A. 1989. Plantas das Caatingas. Rio de Janeiro: Academia Brasileira de Ciências, 1243.

Machado, W.; Prata, A. P.; Mello, A. 2012. Floristic composition in areas of Caatinga and Brejo de Altitude in Sergipe, Brazil. Check List, $\quad 8, \quad 1089-1101$. https://doi.org/10.15560/8.6.1089

Marteis, L. S.; Natal, D.; Sallum, M. A. M.; Medeiros-Sousa, A. R.; La Corte, R. 2017. Mosquitoes of the Caatinga: 2. species from periodic sampling of Bromeliads and tree holes in a dry Brazilian Forest. Acta Tropica, 171 , 114-123. http://dx.doi.org/10.1016/j.actatropica.2017. 03.031

Mori, S. A.; Silva, L. A. M.; Lisboa, G.; Coradin, L. 1985. Manual de manejo de herbário fanerogâmico. Ilhéus: Centro de Pesquisa do Cacau (CEPLAC). 01-104.

Moro, M. F.; Araújo, F. D.; Rodal, M. J. N.; Martins, F. R. 2015. Síntese dos Estudos Florísticos e Fitossociológicos realizados no Semiárido Brasileiro. In: Eisenlohr, P. V. et al. Fitossociologia no Brasil: métodos e 
estudos de caso. Editora da Universidade Federal de Viçosa, pp. 412-451.

Moro, M. F.; Lughadha, E. N.; Araújo, F. S.; Martins, F. R. 2016. A phytogeographical metaanalysis of the semiarid caatinga domain in Brazil. The Botanical Review, 82, 91-148. https://doi.org/10.1007/s12229-016-9164-z

Oliveira, D. G. D.; Prata, A. P. N.; Ferreira, R. A. 2013. Herbáceas da Caatinga: composição florística, fitossociologia e estratégias de sobrevivência em uma comunidade vegetal. Revista Brasileira de Ciências Agrárias, 8, 623-633. https://doi.org/10.5039/agraria.v8i4a2682

Oliveira, E. V. S.; Prata, A. P. N.; Pinto, A. S. 2018. Caracterização e atributos da vegetação herbácea em um fragmento de Caatinga no Estado de Sergipe, Brasil. Hoehnea, 45, 159172. https://doi.org/10.1590/2236-890670/2017

Pereira, F. R. L.; Quirino, Z. G. M. 2008. Fenologia e biologia floral de Neoglaziovia variegata (Bromeliaceae) na Caatinga Paraibana. Rodriguésia, 59, 835-844. https://doi.org/10.1590/2175-

7860200859412

Pontes, R. A. S. 2005. A família Bromeliaceae na Floresta Atlântica da Paraíba, Brasil. Dissertação de Mestrado, Instituto de Pesquisas Jardim Botânico do Rio de Janeiro, Rio de Janeiro, Brasil, 126p.

Queiroz, J. A.; Quirino, Z. G. M.; Lopes, A. V.; Machado, I. C. 2016. Vertebrate mixed pollination system in Encholirium spectabile: a bromeliad pollinated by bats, opossum and hummingbirds in a tropical dry forest. Journal of Arid Environments, 125, 21-30. https://doi.org/10.1016/j.jaridenv.2015.09.01 5

Rocha, F. S.; Duarte, L. S.; Waechter, J. L. 2015. Positive association between Bromelia balansae (Bromeliaceae) and tree seedlings on rocky outcrops of Atlantic forest. Journal of Tropical Ecology, 31, 195-198. https://doi.org/10.1017/S0266467414000728

Sampaio, M. C.; Picó, F. X.; Scarano, F. R. 2005. Ramet demography of a nurse Bromeliad in Brazilian restingas. American Journal of Botany, 92, 674-681. https://doi.org/10.3732/ajb.92.4.674

Santo F. S. E.; Maciel, J. R.; Siqueira Filho, J. A. 2012. Impacto da herbivoria por caprinos sobre as populações naturais de Bromelia laciniosa Mart. Ex Schult. F. (Bromeliaceae). Revista Árvore, 36, 143-149. https://doi.org/10.1590/S010067622012000100015
Scarano, F. R. 2009. Plant communities at the periphery of the Atlantic rain forest: Rarespecies bias and its risks for conservation. Biological Conservation, 142, 1201-1208. https://doi.org/10.1016/j.biocon.2009.02.027

Silva, N. N. F.; Gomes M. L. 2003. Bromeliaceae do Sítio Morro do Céu, Serra (ES). Natureza on line, 1, 1-11.

Silva, A. C. C.; Prata, A. P. N.; Mello, A. A. 2013. Flowering plants of the Grota do Angico Natural Monument, Caatinga of Sergipe, Brazil. Check List, 9, 733-793. https://doi.org/10.15560/9.4.733

Silva, A. C. C.; Prata, A. P. N.; Mello, A. A. 2016. Florística, fitossociologia e caracterização sucessional em um remanescente de Caatinga em Sergipe. Gaia Scientia, 10, 1-14. http://dx.doi.org/10.21707/gs.v10.n04a01

Silva, T. D. S.; Wanderley, M. D. G. L.; Melo, J. I. M. D. 2018. Flora da Paraíba, Brasil: Aechmea Ruiz \& Pav. (Bromeliaceae). Biota Neotropica, 18, e20170401. https://doi.org/10.1590/1676-0611-bn-20170401.

Silvestro, D.; Zizka, G.; Schulte, K. 2014. Disentangling the effects of key innovations on the diversification of Bromelioideae (Bromeliaceae). Evolution, 68, 163-175. https://doi.org/10.1111/evo.12236

Sousa, G. M.; Wanderley, M. G. L. 2015. Bromeliaceae. In: Prata, A. P. N. et al. Flora de Sergipe. Editora Criação, pp.115-142.

Sousa, G. M.; Wanderley, M. G. L. 2000. Achmea Ruiz \& Pav. (Bromeliaceae) do Estado de Pernambuco, Brasil. Acta Botanica Brasilica, 14, 77-97. https://doi.org/10.1590/S010233062000000100008

Sunderland, T.; Apgaua, D.; Baldauf, C.; Blackie, R.; Colfer, C.; Cunningham, A.B.; et al. 2015. Global dry forests: a prologue. International Forestry Review, 17, 1-9. https://doi.org/10.1505/14655481581583481 3

Velloso, A. L.; Sampaio, E. V. S. B.; Pareyn, F. G. C. 2002. Ecorregiões propostas para o bioma Caatinga. Instituto de Conservação Ambiental, 80p.

Versieux, L. M.; Tomaz, E. C.; Jardim, J. G. 2013. New genus and species records of Bromeliaceae in the Caatinga of Rio Grande do Norte state, northeastern Brazil: Orthophytum disjunctum LB Sm. (Bromelioideae) and Tillandsia paraibensis RA Pontes (Tillandsioideae). Check List, 9, 663-665. https://doi.org/10.15560/9.3.663

Versieux, L. M.; Coffani-Nunes, J. V.; Paggi, G. M.; Costa, A. F. 2018. Check-list of 
Bromeliaceae from Mato Grosso do Sul, Brazil. Iheringia, 73, 163-168. https://doi.org/10.21826/24468231201873s 163

Tomaz E. C.; Versieux L. M. 2019. Bromeliaceae from Rio Grande do Norte State, Northeastern Brazil. Phytotaxa, 422, 113-143. https://doi.org/10.11646/phytotaxa.422.2.1
Wanderley, M. G. L. 1990. Diversidade de distribuição geográfica das espécies de Orthophytum (Bromeliaceae). Acta Botanica Brasilica, 4, 169-175. https://doi.org/10.1590/S010233061990000100012 . 